\title{
Tocar o Mirar: Comparación de Procesos Cognitivos en el Aprendizaje con o sin Manipulación Física
}

\section{To Touch or To Watch: Comparison of Mental Processes in the Learning with or without Physical Manipulation}

\author{
Luis Alejandro Andrade Lotero \\ Indiana University, USA \\ Universidad de Cundinamarca, Colombia \\ Derly Rocío Aldana Ahumada
Fundación Tecnológica de Madrid, España
}

\author{
Elquin Antonio Huertas Franco \\ Fundación Tecnológica de Madrid, España \\ Paola Andrea Bacca Pachón \\ Liceo Cervantes Norte, Colombia
}

\begin{abstract}
Resumen. El presente estudio describe los procesos cognitivos que se presentan cuando se aprende a partir de la manipulación física. Para ello, se diseñó un experimento para explorar cómo la utilización de material tangible influye en la resolución de una tarea. Se dividieron 30 niños en dos grupos experimentales. La condición A favoreció la manipulación física debido al uso de un material que los niños podían tocar y mover de un lado al otro para formar agrupaciones. La condición B inhibió la manipulación física debido a que presentó fotos en vez de material tangible, e impidió la agrupación espacial de los materiales. La evidencia empírica muestra que en la condición A los participantes requirieron más tiempo y mayor número de pistas para completar la prueba. A su vez, se encontró que ambas condiciones experimentales fueron igual de efectivas para la consolidación de la información de largo plazo. Se concluye que la presentación del material, más que la manipulación física, ha influido de mayor manera en los procesos cognitivos de los sujetos. Será necesario explorar si otro tipo de actividad, ó disminuir la edad de los sujetos, hace posible que la manipulación física tenga mayor relevancia para el aprendizaje.
\end{abstract}

Palabras clave: aprendizaje, educación, niños, manipulativos, representaciones externas.

\begin{abstract}
This study explores the cognitive processes related to physical manipulation during learning. An experiment was designed to compare the benefits of using tangible materials in a problem-solving task. In this study, we divided 30 children into two experimental groups. Subjects in condition A made use of tangible materials which they could turn, lift, rotate and move to group together. Subjects in condition B were inhibited from learning through physical manipulation, as they used only pictures of the tangible material, and could not group them together because the pictures were all printed on a board. The empirical evidence shows that in condition A subjects required more time and a greater number of hints to complete the task. In addition, we found that subjects in the two conditions were able to recall the characteristics of the groups in the long term. We conclude that the pictorial display of the material rather than physical manipulation was of more influence on the cognitive processes. We suggest using other tasks, or younger children, in order to explore more deeply the importance of physical manipulation during learning.

Keywords: children, education, external representations, learning, manipulatives.
\end{abstract}

Debemos entender que el mundo sólo puede ser comprendido mediante la acción y no a través de la contemplación. La mano es más importante que el ojo... Somos activos; y sabemos con certeza, como algo más que un accidente simbólico en la evolución del hombre, que es la mano la encargada de conducir la evolución subsecuente del cerebro. Hallamos en la actualidad herramientas hechas por el hombre antes de convertirse en tal.

Bronowski, El Ascenso del Hombre

\section{Manipulativos y Aprendizaje}

El presente estudio intenta describir los procesos cognitivos que se presentan cuando se aprende a par-

La correspondencia sobre este artículo debe enviarse al primer autor al e-mail: laandrad@indiana.edu tir de la manipulación física. Para ello, se diseñó un experimento para explorar cómo la utilización de material tangible influye en la resolución de una tarea. Con el objeto de evaluar la influencia de la manipulación física, se contrasta esta prueba frente a la misma tarea utilizando representaciones pictóricas de ese mismo material tangible que no se pueden mover o tocar. Pero, ¿por qué realizar un experimento para comparar el aprendizaje con o sin manipulación física?

Existe un gran recorrido en el uso de materiales tangibles en educación. Una historia breve de los manipulativos incluye a dos personajes, Froebel y Montesori, a quienes se les atribuye el inicio de la educación moderna que propende por el uso de este tipo de materiales (Zuckerman, Arida, y Resnick, 2005). Friedrich Froebel fue el creador del primer jardín infantil, hacia 1837 en Alemania. Froebel diseñó un paquete de 20 
objetos físicos, con el cual los niños podían jugar a reconocer los patrones y formas que se encuentran en la naturaleza. Por su parte, María Montessori desarrolló materiales para niños de mayor edad, pues pensaba que estos manipulativos también juegan un rol importante en los colegios. Ella llamó a esto una "educación de los sentidos", y elaboró actividades y materiales para desarrollar las capacidades sensoriales de los niños. Su objetivo pedagógico era que los manipulativos pusieran al niño en control de su propio aprendizaje, facilitándolo a través de la exploración e investigación personal (Resnick, y otros, 1998).

Desde la teoría constructivista, se ha considerado que el uso de materiales concretos es bueno para el aprendizaje de los niños pequeños. Estos argumentos se basan en la psicología del desarrollo, expuesta por personalidades tales como Piaget (1994), Vygotski (1979) y Bruner (1965). Estos autores proponían que existe un tránsito en el pensamiento que va desde lo concreto hacia lo abstracto, desde lo perceptual hacia lo conceptual. Sin embargo, delimitar qué se debe entender por "concreto" es una cuestión compleja. En realidad, no hay "ideas concretas" en el sentido estricto de la palabra, pues, en esencia, lo concreto hace referencia a lo situado en un contexto específico, pero también a lo tangible, a lo palpable (Clements, 1999). Por su parte, Piaget concebía las primeras etapas del desarrollo en estrecha relación con la realidad perceptible, y los esquemas de acciones como la base para la estructuración cognitiva (Piaget, 1994). A su vez, Vygotsky argumentó la necesidad del aspecto sensorial para el origen del pensamiento (Vygotsky L., 1995). Ambos autores consideraron que las abstracciones, es decir, los procesos de pensamiento que ya no necesitan de un contexto real y tangible, ocurren en una etapa ulterior del desarrollo.

No obstante, a lo largo de las últimas décadas, varios investigadores han elaborado explicaciones alternativas para interpretar los resultados de los experimentos clásicos, así como también han encontrado datos empíricos que apuntan en una dirección diferente (Uttal, Liu, y DeLoache, 2003). En este sentido, se ha mostrado que los niños pequeños pueden exhibir pensamiento abstracto, y, al contrario, también hay evidencia de que los adultos no proceden a partir del pensamiento formal y abstracto cuando intentan resolver problemas en los que son novatos (Uttal, Liu, y DeLoache, 2003).

Ahora bien, con el objetivo de comprender los mecanismos cognitivos que intervienen en el aprendizaje a partir de la manipulación física, aquí se consideran otras teorías que estudian la relación entre lo concreto y lo abstracto. Aunque, de hecho, no es una cuestión de saber cuál aprendizaje es mejor, con o sin manipulación física. Es más bien un problema de comprender los procesos cognitivos que suceden en la mente de un sujeto cuando se apoya en la manipulación física para realizar una tarea.
Así pues, se ha recurrido a la ciencia cognitiva y a las diversas investigaciones acerca de los mecanismos cognitivos que están involucrados en el aprendizaje. En este sentido, la ciencia cognitiva ha estudiado el procesamiento de información que se produce tanto dentro de la mente como representación interna, como aquel que se apoya en artefactos culturales y exterioriza la cognición como "representación externa" (Scaife y Rogers, 1996). Estos artefactos pueden ser objetos materiales, gráficos, símbolos, y otros recursos nemotécnicos que apoyan la manera como el ser humano procesa, interpreta y representa la información (Gauvain, 2001). Entonces, ¿qué constructos teóricos provenientes de la ciencia cognitiva pueden predecir los resultados del aprendizaje por medio de la manipulación física?

\section{Cognición y manipulación física}

En el presente trabajo se considerarán cuatro constructos teóricos, los cuales se han sintetizado en una corta descripción que no pretende ser exhaustiva, ni desconocer otros enfoques de estos mismos. A continuación se describen brevemente las ideas de: 1) carga cognitiva, 2) mapeo cognitivo, 3) representación dual, y 4) pericia, así como su relación con el aprendizaje apoyado en la manipulación física.

\section{Carga Cognitiva}

En la literatura se habla de "descarga cognitiva" como el proceso por medio del cual se liberan recursos en la memoria de trabajo de los sujetos. La premisa básica es que los aprendices tienen una muy limitada capacidad de memoria de trabajo cuando deben aprender nueva información. El aprendizaje se verá amenazado si los materiales instruccionales sobrecargan los limitados recursos de la memoria de trabajo del aprendiz (Artino, 2008; Sweller, 2002). Según la teoría, existen tres tipos de carga cognitiva: a) intrínseca, b) extrínseca y c) pertinente. La carga cognitiva intrínseca se refiere a la complejidad de la tarea y la experticia del sujeto. La extrínseca es el exceso de carga producido por un mal diseño instruccional. Finalmente, la carga cognitiva relevante ayuda a generar y automatizar esquemas en la memoria de largo plazo (Sweller, 2002).

Con respecto a la manipulación física, la información proveniente de la disposición espacial de los objetos permite a los sujetos llevar un registro de la posición de los mismos, liberando así la atención visual a otros objetos. De esta manera, se reducen las demandas en la memoria viso-espacial, se ayuda a recuperar información y a recordarla. Cuando el problema es demasiado difícil, la manipulación física ayuda liberando recursos cognitivos en forma de andamiaje 
(Manches y O'Malley, in press). Sin embargo, también es necesario considerar que demasiada descarga cognitiva no contribuye al aprendizaje, pues lo que consigue es que el sujeto realice un procesamiento superficial de la información (Kalyuga, Ayres, Chandler, y Sweller, 2003; van Nimwegen, Burgos, van Oostendorp, y Schijf, 2006).

\section{Mapeo Cognitivo}

También se ha hablado de "metáforas cognitivas" o "razonamiento analógico". El proceso de mapeo cognitivo se refiere a la capacidad de entender una idea, de apropiar un dominio conceptual, por medio de otra idea perteneciente a un dominio diferente. La literatura también lo entiende como una analogía entre un "dominio de origen", el entorno físico, y un "dominio de llegada", en forma de conceptos abstractos (Manches y O'Malley, in press). Una muestra de ello es por ejemplo hablar de "la evolución biológica como si fuera un árbol con muchas ramas", o "el devenir del tiempo como si fuera un río". Comprender los conceptos implica establecer proyecciones metafóricas entre la información del entorno y la formación de esquemas mentales que representan internamente esa información (Ainsworth S., 2006; Manches y O’Malley, in press; Núñez, Edwards, y Matos, 1999).

Según Clements (1999), las ideas que representan los materiales tangibles son igual de abstractas que los símbolos que representan. En este sentido, los manipulativos por sí mismos no son lo que importa, sino la manera como ayudan a relacionar las ideas, a integrar lo concreto con lo abstracto. Esta manera de entender lo concreto fue denominada por Clements como "concreto integrado".

\section{Representación Dual}

El pensamiento simbólico implica que el sujeto puede entender un objeto como la representación de otra cosa. En este sentido, el objeto que sirve de representación tiene un carácter dual, pues es a la vez objeto y representación. En ocasiones, las propiedades del objeto pueden afectar, ya sea facilitando o dificultando, que un sujeto pueda tomarlo como algo más, es decir, como un símbolo (Uttal, Liu, y DeLoache, 2003). El estudio del desarrollo del pensamiento simbólico en los niños ha hecho interesantes descubrimientos frente al uso de materiales tangibles y su representación como símbolos. Según varios autores (Uttal, Scudder, y DeLoache, 1997), un buen manipulativo debe facilitar la doble representación. No obstante, los objetos que resaltan y tienen características llamativas en sí mismos pueden no llegar a ser buenos manipulativos, porque impiden ser vistos como símbolos. Así pues, representaciones menos "concretas", como fotografías de objetos, pueden llegar a mejorar el desempeño de los niños pequeños (Uttal, Liu, y DeLoache, 1999). En estos casos, disminuir la atención en el objeto mismo puede aumentar la posibilidad de que sea visto como un símbolo.

\section{Pericia}

Los estudios sobre el desarrollo de las habilidades en la resolución de problemas plantean que éste es un proceso de aumento cuantitativo de conocimiento de dominio (Thornton, 1998). Los estudios de resolución de problemas destacan que los expertos, en contraposición a los novatos, son capaces de reconocer características y patrones de las situaciones novedosas. Los novatos, por su parte, dedican toda su concentración a entender las situaciones inmediatas puesto que su conocimiento es fragmentado y desconectado (Wood, 2000). Con el tiempo y la práctica, se automatizan los esquemas que permiten organizar mejor la información, discernir una estructura y hacer predicciones. En este sentido, las investigaciones predicen que aprender a partir de objetos manipulativos no es algo que suceda espontáneamente, sino todo lo contrario. A los niños se les debe enseñar a leer los manipulativos; y esto es un proceso que requiere de apoyo constante para lograr que lleguen a relacionar las representaciones externas con el concepto abstracto que representan (Ainsworth S., 2008; Clements, 1999; Uttal, Liu, y DeLoache, 1999).

\section{Explorando la cognición y la manipulación física}

¿Qué hipótesis surgen al planear un experimento que pretende comparar el aprendizaje con o sin manipulación física?

Por un lado, es evidente que para la psicología del desarrollo los materiales tangibles ayudan al aprendizaje, puesto que son concretos y los niños se encuentran en una etapa de pensamiento concreto. Además, según Bruner (1965), los objetos físicos facilitarían el aprendizaje a partir de lo perceptual, en comparación con un material que imponga necesidades más conceptuales o abstractas, como las representaciones pictóricas o aquellas que no facilitan la manipulación.

En contraste, según la ciencia cognitiva, se pueden extraer conjeturas diferentes:

Según la teoría de la carga cognitiva, los tangibles ayudan en la descarga cognitiva, pero deben estar diseñados de tal manera que ayuden a disminuir la carga cognitiva extraña, es decir, aquella que proviene de un mal diseño instruccional (Sweller, 2002). Asimismo, si la tarea no es lo suficientemente compleja, es posible que se presente lo que se ha denominado el "efecto inversión", debido a un exceso de andamiaje (Kalyuga, Ayres, Chandler, y Sweller, 2003). 
Según las teorías del mapeo cognitivo, los tangibles pueden favorecer la representación de la información, pues la adaptación de las representaciones puede ayudar físicamente al aprendizaje (Martin y Schwartz, 2005). En este sentido, la disposición espacial de los objetos puede ayudar a procesar información y a servir de puente entre las ideas abstractas y las representaciones externas.

Según la teoría de la representación dual, si los tangibles son muy llamativos en sí mismos, estos no ayudarán al proceso de simbolización de los niños pequeños (Uttal, Liu, y DeLoache, 2003).

Según la teoría de la pericia, la manera como los manipulativos representan los conceptos abstractos es un proceso que requiere práctica y tiempo. "El conocimiento no viaja desde los dedos subiendo por el brazo hasta la cabeza" (Clements, 1999, pág. 7). Aprender con los manipulativos requerirá de un apoyo constante para reflexionar acerca de cómo se relacionan las representaciones físicas con las ideas abstractas.

\section{Estudiando la manipulación física durante el aprendizaje}

Un experimento interesante donde se ponen a prueba los manipulativos y el proceso de simbolización es el experimento clásico realizado por Vigotsky (1995), conocido como el Método de la Doble Estimulación. Este experimento consistía en 22 cubos de madera con 5 formas diferentes, 5 colores, 2 grosores y 2 tamaños. El objetivo era descubrir una "palabra oculta", la cual no tiene significado en ninguna lengua, a partir de la agrupación, primero aleatoria y luego más ordenada de los materiales, conforme aumenta la información disponible a lo largo de la prueba.

Según Vygotsky (1995), la formación de conceptos implica un proceso creativo donde se establecen relaciones y se categoriza la realidad. Vygotsky utilizó esta herramienta para medir el proceso de formación de conceptos a lo largo de diversos estadios. En este trán- sito, la actividad y la palabra son igualmente importantes. La actividad representa el pensamiento en sus niveles iniciales (representa de manera dinámica lo que el sujeto está pensando), mientras que la segunda (la palabra) actúa como medio y luego como símbolo del concepto, es decir, cumple una función reguladora del pensamiento.

En una investigación anterior de tipo cualitativodescriptivo realizada por el grupo de investigación (2011), se adaptó la prueba original de Vygotsky para ser trabajada con niños entre los 6 y los 8 años. En tal adaptación, los trozos de madera fueron reemplazados por materiales tangibles de la cotidianidad de los niños. Tres tipos de materiales fueron construidos: muñecas, carros y casas (ver figura 1).

Durante el análisis de resultados se evidenció que la formación de conceptos no fue homogénea con respecto a la edad. Además, se encontró que los sujetos de 8 años eran más minuciosos y observadores que los de 6 años, por lo que tomaron más tiempo para clasificar los objetos. Los manipulativos físicos brindaron a los niños la oportunidad de tocar, coger, mover, trasladar y organizar su material de aprendizaje. De esta manera, se les facilitó observar las características de los objetos y los detalles de los mismos. Un aspecto que emergió de tal investigación y que vale la pena destacar fue con respecto al género. Se encontró que en estos experimentos era más fácil que las niñas quisieran jugar con los carros antes que los niños con las muñecas (2011). Por ello, en las experiencias posteriores se ha utilizando exclusivamente el material de las casas, el cual no plantea sesgos de género.

Pero, ¿cómo responderá este material ante la comparación con una representación pictórica que inhiba la manipulación física? ¿Cómo interpretar los resultados a la luz de la teoría arriba discutida?

A continuación, se presenta un experimento diseñado para medir las diferencias en los procesos de aprendizaje comparando dos situaciones: 1) en la cual se favorece aprender a partir de la manipulación física de los objetos tangibles que componen el material instruc-

Figura 1. Material tangible creado para adaptar la prueba de la doble estimulación de Vygotsky
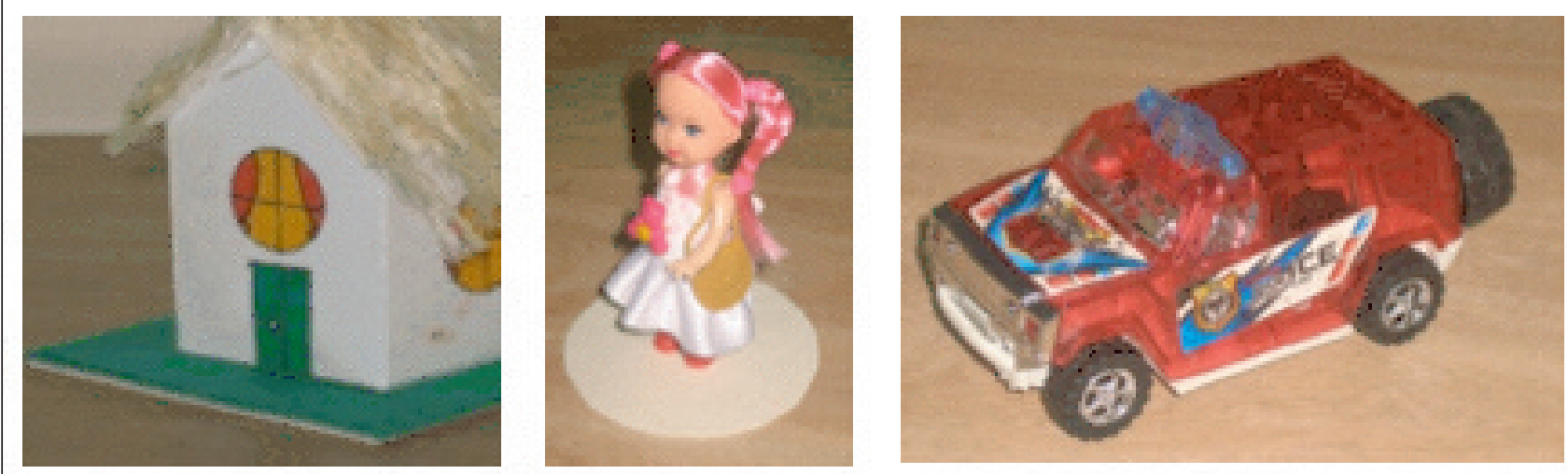
cional; y 2) en la cual se impide aprender a partir de la manipulación física.

\section{Metodología}

\section{Participantes}

Los participantes en el estudio empírico fueron estudiantes de sexo masculino de $2^{\circ}$ y $3^{\circ}$ de primaria, entre los 7 y los 9 años. Los sujetos pertenecen a un colegio privado de la ciudad capital y cuyo nivel socio económico está en los estratos 4 y 5. Es importante tener en cuenta que ninguno de estos sujetos ha participado antes en estudios experimentales.

La muestra estuvo constituida por 30 niños $(M=8.4$ años y $D S=0.69$ años) del mismo colegio, escogidos al azar y divididos en dos grupos. Se asignaron 15 estudiantes al grupo A de objetos tangibles $(M=8.6$ años $D S=0.72)$ y 15 sujetos al grupo B de representaciones pictóricas $(M=8.3$ años $D S=0,67)$.

\section{Diseño experimental}

El diseño experimental propuesto plantea dos grupos apoyados en el mismo protocolo de intervención. En la condición A (ver figura 2), los niños hicieron uso de materiales tangibles, y en la condición B (ver figura 3) los niños hicieron uso de representaciones pictóricas idénticas a los materiales tangibles. En el grupo $\mathrm{A}$, los participantes pudieron observar, tocar y manipu-

Figura 2. Condición experimental A: representaciones tangibles

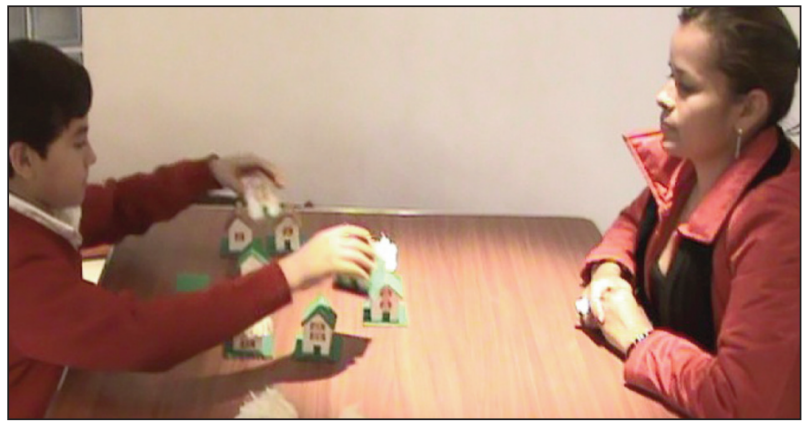

Figura 3. Condición experimental B: representaciones pictóricas

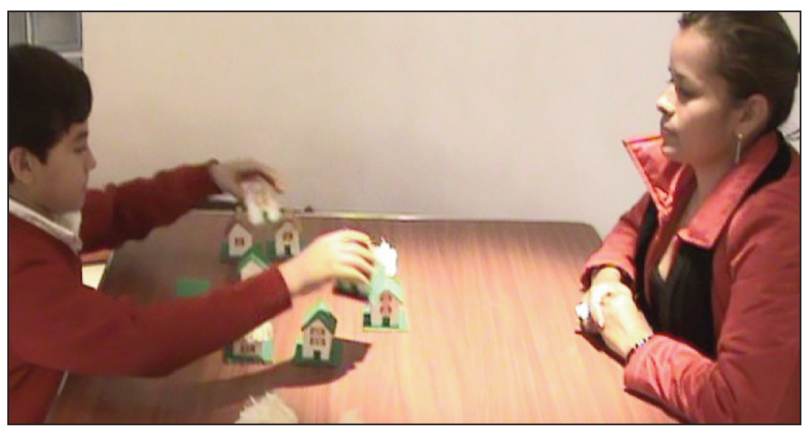

lar el material. Por su parte, los sujetos del grupo B sólo pudieron observar un tablero acrílico con las fotos del material tangible y utilizar un marcador para desarrollar la tarea.

Todas las variables importantes tales como la instrucción y los tipos de preguntas por parte del tutor, fueron las mismas en ambas condiciones.

\section{Materiales}

Para la condición tangible se elaboraron 12 casas de $6,5 \mathrm{~cm}$ de alto x $7 \mathrm{~cm}$ de largo, construidas en cartón paja, fomi, teja de cerámica y paja (ver figura 4). Luego, para la condición pictórica se tomaron fotos de esas 12 casas y se imprimieron en un tablero acrílico de $70 \mathrm{~cm}$ de largo $\mathrm{x} 48 \mathrm{~cm}$ de ancho. Es importante anotar que las fotografías de las casas tienen la misma medida que las casas tangibles. El tablero está protegido con una lámina de acetato del mismo tamaño, con el fin de permitir que el sujeto pueda marcar las imágenes pictóricas con un marcador borrable.

Figura 4. Representaciones Tangibles y Atributos utilizados en el experimento

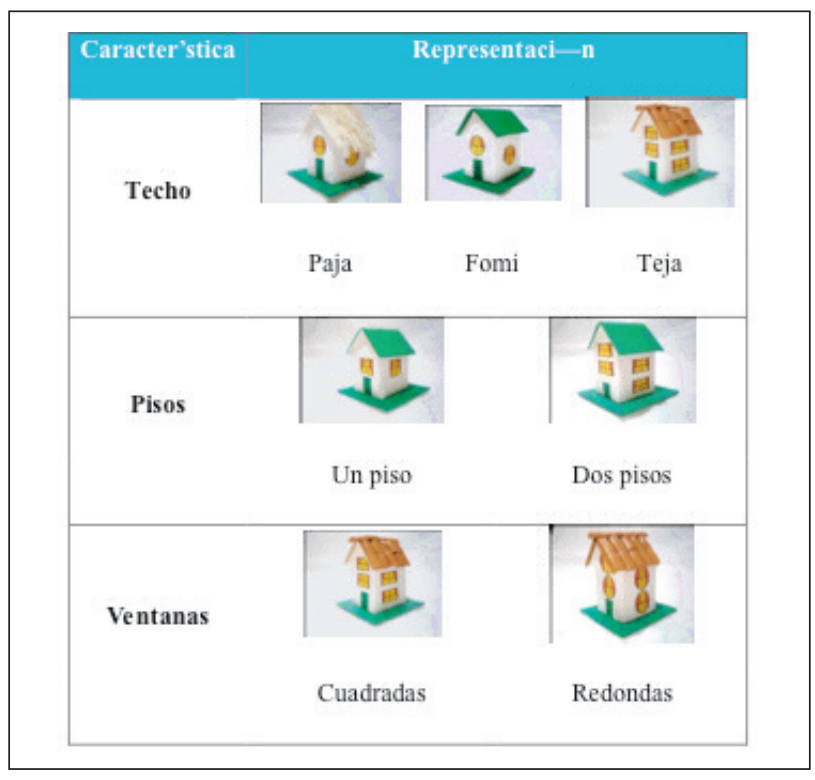

\section{Post-test}

Se diseñó una prueba con la cual se midió el nivel de retención de conocimientos de los participantes de ambos grupos. La prueba consta de 6 preguntas, cada una de las cuales muestra tres fotografías de casas, una correcta y dos incorrectas. La prueba fue aplicada a los sujetos de ambos grupos dos meses después de la intervención. Para el desarrollo de la prueba se asignó un salón de clase con buena iluminación y puestos individuales a los estudiantes, y estuvo orientada por uno de los investigadores. 


\section{Procedimiento}

El experimento compara la eficacia del uso de manipulativos tangibles frente a fotos como representaciones pictóricas de esos mismos manipulativos, las dos condiciones de aprendizaje se diferencian sólo en el medio de presentación. El experimento consistió en descubrir varias "palabras ocultas" a través de un juego. En total, fueron tres palabras que cada sujeto debía descubrir. La primera palabra oculta (Curtem) correspondió al atributo techo de paja; para la segunda palabra (Dactu) se utilizó el atributo casas de dos pisos; y para la tercera palabra (Mirka) el atributo fue ventanas cuadradas.

Se elaboraron tres protocolos de intervención, uno para cada palabra oculta. Cada protocolo consistía en una serie de pautas para interactuar con el sujeto, así como una serie de pistas para ayudar a descubrir las palabras. Las pistas estaban compuestas por dos ejemplos y un contraejemplo, lo que representaba el número de ayudas que el participante podía utilizar para resolver la tarea. El último tipo de ayuda, en caso de que el sujeto aún no pudiera resolver el problema, fue explicar cómo se agrupaban los materiales para darle sentido a la palabra oculta. El protocolo permitió tener unas instrucciones claras, adecuadas y oportunas con los participantes.

Se escogió un espacio luminoso, aislado del ruido y acondicionado con una mesa y dos sillas. Adicionalmente, se contaba con una cámara de video en trípode, lo que permitió tener un registro visual de los estudiantes mientras resolvían la tarea. Primero se realizó el experimento con el grupo A de representaciones tangibles. Terminado el experimento con este grupo, se prosiguió con el grupo B en un tablero acrílico. El experimento fue similar al anterior, sin embargo, el sujeto en lugar de manipular las casas, debía subrayar con un marcador borrable sobre el tablero acrílico, dependiendo de la palabra que iba a descubrir.

Finalmente, se midieron las siguientes características obtenidas individualmente para cada sujeto:

a) Tiempo en la tarea: se midió el tiempo transcurrido desde que se dio la primera pista hasta que respondió correctamente cada palabra oculta. b) Número de pistas: Se contó el número de pistas, compuestas por ejemplos y contraejemplos, que empleó el aprendiz en total para descubrir el significado del concepto artificial (las cuales podían ir de $1 \mathrm{a} 4)$.

c) Retención y consolidación de conocimientos: prueba que midió la conformación de esquemas en la memoria de largo plazo. Esta se realizó dos meses después de aplicada la prueba.

\section{Resultados}

Se utilizó la Prueba U de Mann-Whitney para analizar los resultados. Esta prueba no paramétrica se utiliza para comparar la diferencia entre dos muestras independientes, y es usada frecuentemente en situaciones donde las dos muestras provienen de la misma población pero cada grupo recibe una intervención diferente (Robert, 1996). Los resultados obtenidos en cada condición experimental se presentan en la tabla 1.

\section{Tiempo en la tarea}

El grupo que utilizó manipulativos tangibles reportó un promedio de $3: 54 \mathrm{~min}(D S=72.4 \mathrm{seg})$. El grupo que trabajó con representaciones pictóricas tuvo un promedio de 2:36min ( $D S=48 \mathrm{seg})$. Se concluye que existe suficiente evidencia, con un nivel de confianza de $=0.05$, de que existe una diferencia significativa entre las dos muestras $(p=0.002)$. Es decir, los sujetos que utilizaron las representaciones pictóricas gastaron menos tiempo en la tarea que los que trabajaron con el material tangible.

\section{Número de pistas}

El grupo que utilizó las representaciones pictóricas tuvo un promedio de 2.0 pistas $(D S=0.42)$. El grupo de representaciones tangibles reportó un promedio de 2,4 pistas $(D S=0.53)$. Se concluye que existe suficiente evidencia, con una confianza de $=0.05$, de que

Tabla 1. Datos obtenidos en las dos condiciones experimentales de la prueba

\begin{tabular}{|c|c|c|c|c|}
\hline \multirow{4}{*}{ 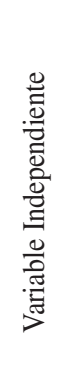 } & \multicolumn{4}{|c|}{ Variable dependiente } \\
\hline & & o en la tarea(minutos) & Número de pistas & Nivel de retención \\
\hline & $\begin{array}{c}\text { Condición A: } \\
\text { Representaciones } \\
\text { Tangibles }\end{array}$ & $\mathbf{3 : 5 4}(d s=1: 12)$ & $\mathbf{2 , 4}(d s=0,42)$ & $\mathbf{0 , 6 1}(d s=0,19)$ \\
\hline & $\begin{array}{c}\text { Condición B: } \\
\text { Representaciones } \\
\text { Pictóricas }\end{array}$ & $\mathbf{2 : 3 6}(d s=0: 48,8)$ & $\mathbf{2 , 0}(d s=0,53)$ & $\mathbf{0 , 6 7}(d s=0,2)$ \\
\hline
\end{tabular}


existe una diferencia significativa entre las dos muestras $(p=0.039)$. Es decir, los sujetos que trabajaron con las representaciones pictóricas utilizaron un menor número de pistas para realizar la tarea que los que trabajaron con las representaciones tangibles.

\section{Nivel de Retención}

Según la prueba de retención, los estudiantes que trabajaron con las representaciones pictóricas tuvieron un puntaje de $0.67(D S=0.20)$. Por su parte, para el grupo de manipulación tangible la media es de $0.61(D S=0.19)$. Se concluye que, tomando una confianza de $=0.05$, no existe suficiente evidencia para concluir que existe una diferencia significativa entre las dos muestras $(p=0.337)$. Es decir, los niños que trabajaron con las representaciones pictóricas obtuvieron el mismo nivel de retención que los que trabajaron con las representaciones tangibles. No hay diferencia significativa en la retención de los conceptos.

\section{Descripción del desempeño}

Se puede anotar que los materiales, tanto tangibles como pictóricos, favorecieron las conductas de exploración propias de la edad de los niños con los que se realizó la prueba. Todos los sujetos entendieron el objetivo del juego, mas no utilizaron los manipulativos para inventarse juegos de imaginación que no tuvieran que ver con la prueba. Por ejemplo, pensar que las casas formaban un conjunto residencial donde vivían sus amiguitos del colegio, etc. A pesar de que el material tangible es llamativo por sus características, los niños se limitaron a cumplir la instrucción dejando de lado la posibilidad de jugar con este. Probablemente, esto se debe a la disposición de los niños para seguir instrucciones y la etapa de desarrollo en la que se encuentran.

Ahora bien, como se muestra en la figura 5, en promedio los niños que utilizaron el tablero tomaron menos tiempo para dar sus respuestas en comparación con el material tangible. No obstante, también es posible apreciar que, luego de cada prueba, el tiempo fue aumentando para la condición tablero, mientras que disminuyó casi un minuto para la condición tangible. Así pues, en la prueba 3, el tiempo es casi equiparable para ambas condiciones. Si esta tendencia se mantuviera, cabe entonces preguntarse si en intentos sucesivos se acabaría la diferencia entre el material tangible y el pictórico, o acaso podría llegar a ser menor para este primero.

A su vez, es interesante notar que el número de pistas en la prueba 3 para el material tangible fue casi igual de bajo a los que usaron el tablero (ver figura 6). Aunque la tendencia general es que el material tangible requirió mayor número de pistas, durante el último
Figura 5. Tiempo en la tarea discriminado por cada una de las tres pruebas realizadas a cada sujeto

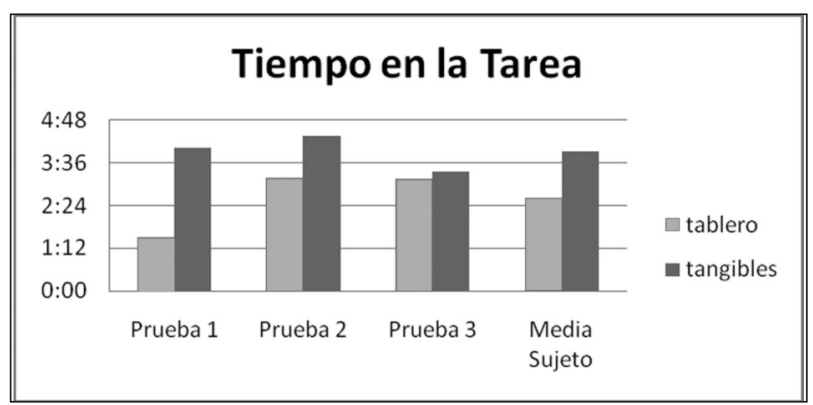

Figura 6. Número de pistas discriminado por cada una de las tres pruebas realizadas a cada sujeto

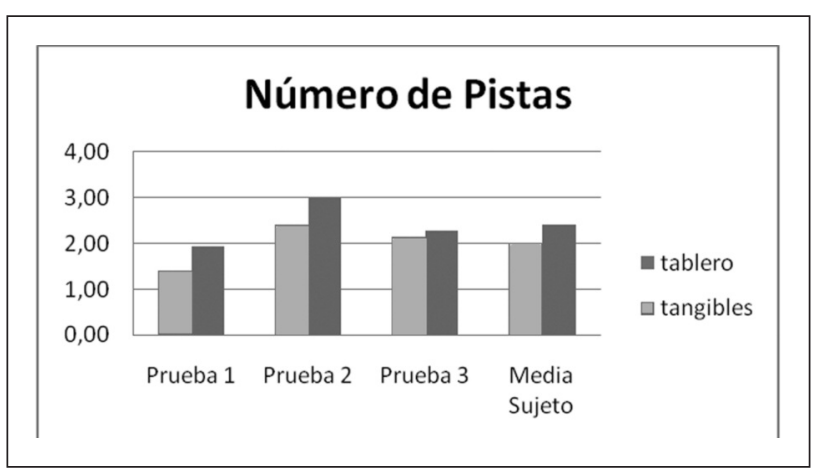

Figura 7. Puntaje en la prueba de retención discriminado por cada pregunta realizada a cada sujeto

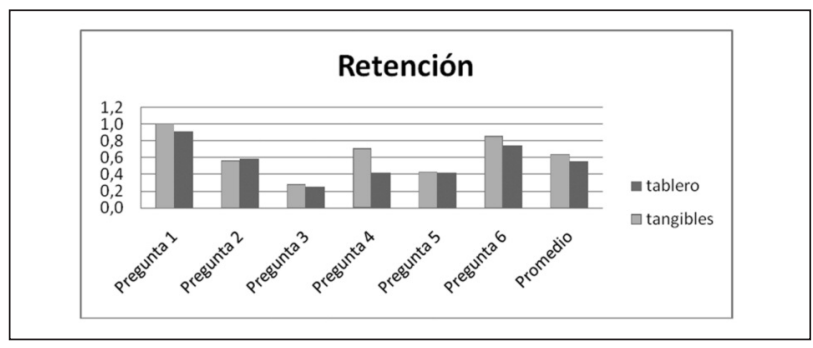

intento parece haber poca diferencia. Cabe preguntarse si se mantuviera esta tendencia, si en intentos posteriores el número de pistas para ambos materiales puede ser el mismo.

Frente a la prueba de retención, es evidente que sistemáticamente los promedios son mayores para el material tablero, pero la diferencia es muy poca (ver figura 7). La única diferencia sensible corresponde a la pregunta 4 , en donde el puntaje del grupo tablero parece duplicar al puntaje del grupo tangible. Además, es curioso que el $100 \%$ de los sujetos del grupo tablero respondiera acertadamente a la pregunta 1 .

\section{Conclusiones}

A continuación se discute cómo se relacionan las características medidas con respecto a los conceptos provenientes de la ciencia cognitiva arriba presentados. 


\section{Tiempo en la tarea}

Evidentemente, la interacción con los materiales físicos implica mayor tiempo en la tarea. No fue lo mismo trazar una equis encima de una foto con un marcador, a mover doce casas de un lado para otro sobre una mesa; incluso el tiempo de borrar las marcas cuando el niño se equivocaba no se equipara con volver a acomodar todo el material tangible en el centro de la mesa.

Ahora bien, también puede estar directamente relacionado con el nivel de carga cognitiva, pues a mayor carga cognitiva mayor tiempo en la tarea (Tabbers, Martens, y van Merriënboer, 2004). Asimismo, un posible problema es el llamado efecto representación dual, en donde el objeto no es concebido como una representación sino como un objeto en sí mismo (Uttal, Liu, y DeLoache, 1999; Uttal D. H., 2003; Uttal, Scudder, y DeLoache, 1997). En este sentido, el efecto dual podría afectar el tiempo que gasta el aprendiz en percibir que los manipulativos son una representación de una agrupación por un atributo común que tiene como significante una palabra artificial.

La diferencia significativa en el tiempo podría indicar que los niños que utilizaron el tablero se demoraron menos en resolver la tarea debido a que tuvieron mayor facilidad para observar las características pertinentes de los objetos. Es posible que los manipulativos tangibles sean más llamativos para los niños, y esto hace que generen distracción al momento de manipularlos para realizar la tarea que se les pide. En resumen, manipular el material tangible llevó a los niños a utilizar más tiempo, pero no les facilitó encontrar los atributos que los diseñadores de la prueba seleccionaron, tales como la forma de las ventanas, el tipo de material del techo y el número de pisos de las casas. Por el contrario, los niños que jugaron con el material pictórico eligieron más rápidamente los atributos que podían tener significado para la palabra oculta.

\section{Número de pistas}

El nivel de apoyo que recibe el aprendiz puede estar relacionado con la carga cognitiva de tipo extraña (Artino, 2008; Sweller, 2002), la cual podría estar interfiriendo en la atención del sujeto y su habilidad para organizar y clasificar la información. Los niños que jugaron con las casas tangibles requirieron más ayuda para encontrar el significado de la palabra oculta, posiblemente porque la dificultad intrínseca del material es más alta que en las representaciones pictóricas. En este sentido, según la teoría de la carga cognitiva, la carga cognitiva intrínseca es mayor para el material tangible y, a su vez, la carga cognitiva extrínseca es inferior en el material pictórico. Es decir, con las fotos fue más fácil reconocer los elementos de significado y la manera como estos elementos se relacionan los unos con los otros.
A su vez, la dificultad para concebir a los materiales tangibles como símbolos de una palabra oculta, que progresivamente se va descubriendo, apoya la teoría de la representación dual. Las casas tangibles son muy llamativas en sí mismas, y requirieron más apoyo para que tomaran significado como símbolo de la palabra oculta.

\section{Retención y consolidación de conocimientos}

Al no haber diferencia significativa entre las dos condiciones experimentales, es posible concluir que no hay evidencia para afirmar que la manipulación física beneficia o perjudica la formación de esquemas en la memoria de largo plazo. Tanto los niños con las casas tangibles como los niños con las fotos, recordaron muy bien el significado de las palabras artificiales. A su vez, se podría decir que las metáforas contenidas en la distribución espacial de los grupos de casas tangibles no contribuyeron a mejorar la consolidación del nuevo conocimiento. No importó si los niños pudieron reunir físicamente (en todo el sentido de la palabra agrupar) las casas que pertenecían al atributo de la palabra artificial. De la misma manera, las marcas realizadas sobre las fotos fueron suficientemente claras para que los niños pudieran recordar los atributos.

Finalmente, es posible anotar que la media de retención en ambos grupos estuvo muy por encima del nivel de azar, y ello puede implicar que para esta población este tipo de tarea es relativamente fácil. En otras palabras, la prueba con 12 casas y con tres características diferentes no provee una fuerte exigencia cognitiva, o dicho de otro modo, no tiene un efecto techo adecuado.

\section{Discusión}

El objetivo fue comparar los efectos de la manipulación física en el aprendizaje. La condición experimental A favoreció la manipulación física debido al uso de un material tangible que los niños podían tocar, levantar, rotar, y esencialmente, mover de un lado al otro para formar agrupaciones. La condición experimental B inhibió la manipulación física debido a que presentó fotos en vez de material tangible, y restringió la agrupación espacial de los materiales reemplazándolo por marcas realizadas con un marcador.

La evidencia empírica sugiere que los participantes en la condición experimental A requirieron más tiempo y un mayor número de ayudas para completar la prueba. A su vez, se encontró que ambos materiales fueron igual de efectivos para la consolidación de la información de largo plazo. Al comparar los procesos cognitivos se puede concluir que el material en la condición B le permitió al participante utilizar con mayor efectividad su memoria viso-espacial. Por su parte, manipular 
el material tangible de cierta manera generó distracción, y esto hizo que su atención y memoria disminuyeran a la hora de desarrollar la prueba.

El resultado obtenido para la condición experimental B evidencia que, aunque son representaciones menos concretas, permitieron un mejor desempeño en este tipo de prueba. El uso de las fotografías en el tablero acrílico resultó ser una adecuada representación simbólica que hizo las veces de objeto y de representación. También se puede evidenciar que los manipulativos tangibles son más llamativos para los niños y esto hace que generen cierta distracción al momento de manipularlos para realizar la tarea que se les pide.

No obstante, conforme pasaban de una prueba a la otra, los sujetos parecen desempeñarse más homogéneamente en cuanto al tiempo y al número de pistas empleado. Es posible resaltar que en el último intento (prueba 3 ), estas dos variables son muy similares entre ambas condiciones experimentales. En efecto, es posible que conforme aumentaba la experiencia de los sujetos frente a la prueba, mayor era la probabilidad de que su desempeño fuese similar no importa qué material utilizaran. No obstante, estos datos necesitan más investigación, ya que sólo son tendencias que parecen presentarse.

En conclusión, se puede señalar que los constructos provenientes de la ciencia cognitiva tienen un valor teórico para explicar el aprendizaje por medio de material concreto y manipulativo. En general, es posible decir que el diseño de material instruccional debe tener en cuenta los procesos cognitivos aquí descritos: carga cognitiva, mapeo cognitivo, representación dual, y pericia. Al momento de explicar la evidencia empírica, este marco teórico puede predecir el desempeño de los niños, a diferencia de las teorías clásicas del desarrollo cognitivo. Según estas últimas, el uso de lo "concreto" es beneficioso para niños pequeños, quienes se encuentran en un nivel de pensamiento también concreto.

Por su parte, no ha sido posible encontrar algún efecto de la manipulación física en el aprendizaje de los niños. Es cierto que permitir la actividad física con objetos tangibles aumentó el tiempo en la tarea, lo cual puede ser beneficioso para el aprendizaje. Sin embargo, también se encontró la necesidad de realizar un mayor apoyo para que los niños construyeran sentido a las representaciones. Finalmente, si la consolidación de la información en la memoria se da en el mismo grado en ambas condiciones experimentales, se podría asumir que la manipulación física no es una variable que permita mejorar el aprendizaje en esta población de niños y para este tipo de actividad.

\section{Sugerencias y recomendaciones para nuevas investigaciones}

Es muy importante considerar las características de la población de estudio, como el nivel socio económico o el desarrollo cognitivo, pues son factores determinantes para analizar los procesos cognitivos de los niños. Es posible que variar alguna de estas variables conlleve ciertas repercusiones frente al desempeño en este tipo de pruebas. Además, cuando se hacen comparaciones con diversos materiales, es importante controlar su elaboración así como las indicaciones para el uso de los mismos. En este caso, se recomienda tener en consideración el ángulo en el cual se toman las fotografías de los objetos, ya que pueden alterar el desarrollo de la prueba.

Por último, para posteriores investigaciones será necesario ampliar el tipo de actividades para estudiar la manera como la manipulación física puede afectar el aprendizaje. Además, es posible que si se disminuye la edad de los sujetos la variable manipulación física podría tener alguna relevancia. Hasta el momento, ha sido más importante prestar atención a la forma de presentación del material, más que al tipo de interacción con el mismo.

\section{Extended Summary}

The present study attempts to describe the cognitive processes that occur when we learn from physical manipulation. To do this, we designed an experiment to explore how the use of tangible material affects the resolution of a task. In order to evaluate the influence of physical manipulation, this test is contrasted against the same task using pictorial representations only of the same tangible material, which cannot be moved or touched. However, first it is necessary to answer the question of why it is important to compare learning with physical manipulation and that without.

There is a great history of the use of tangible materials in education. A brief history of manipulatives must include two characters, Froebel and Montessori, to whom the beginning of modern education aiming for the use of these materials is attributed (Zuckerman,
Arida, \& Resnick, 2005). From the point of view of constructivist theory, it is considered that the use of concrete materials is beneficial in the teaching of young children. These arguments are based on developmental psychology, as stated by people such as Piaget (1994), Vygotsky (1979) and Bruner (1965).

However, over recent decades, researchers have developed several alternative explanations for interpreting the results of classic experiments, and also found evidence pointing in a different direction (Uttal, Liu, \& DeLoache, 2003). Now, with the aim of understanding the cognitive mechanisms involved in learning from physical manipulation, other theories are considered here to study the relationship between the concrete and the abstract.

In this paper, we considered four theoretical con- 
structs: 1) cognitive load, 2) cognitive mapping, 3) dual representation, and 4) expertise, and their relationship to learning supported by physical manipulation.

What hypotheses arise when planning an experiment that seeks to compare learning physical manipulation and learning without it? On the one hand, it is clear that developmental psychology considers that tangible materials help learning, since children are in a stage of concrete thinking. On the other hand, according to cognitive science, different assumptions can be drawn:

According to cognitive load theory, tangibles help in cognitive download, but must be designed so as to help reduce the foreign cognitive load, i.e. that which comes from a poor instructional design (Sweller, 2002). According to theories of cognitive mapping, tangibles can promote the representation of information, since adapting physical representations can help support learning (Martin \& Schwartz, 2005). According to dual representation theory, if the tangibles are very striking in themselves, they do not help the process of symbolization for young children (Uttal, Liu, \& DeLoache, 2003). According to the theory of expertise, making manipulatives represent abstract concepts is a process that takes practice and time (Clements, 1999).

In a previous qualitative-descriptive study (Andrade Lotero et al., 2011), we adapted the original Vygotsky (1995) Double Stimulation Method for use with children between 6 and 8 years old. In this adaptation, the wood pieces were replaced with tangible materials from children's every day life. Three types of materials were constructed: dolls, cars and houses

\section{Method}

We performed an experiment designed to measure differences in the learning process by comparing two situations: 1) encouraging learning from the physical manipulation of tangible objects that make up the instructional material, and 2) precluding learning from physical manipulation.

In this empirical study, the participants were male students of 2nd and 3rd grade, between 7 and 9 years old. The subjects belong to a private school in the capital city, and in which their socio-economic status is in strata 4 and 5 . The sample consisted of 30 children $(M$ $=8.4$ years, $S D=0.69$ years) from the same school, chosen at random and divided into two groups. Fifteen students were assigned to group A, using tangible objects $(M=8.6$ years $S D=0.72)$ and fifteen subjects in group $\mathrm{B}$, with only pictorial representations $(M=8.3$ years $S D=0.67)$.

The experimental design was based on the same intervention protocol. In condition A (see Figure 2), the children made use of tangible materials, and in condi- tion B (see Figure 3), children made use of pictorial representations identical to the tangible material. In group A, participants were able to observe, touch and manipulate the material. The children in group B, however, only observed an acrylic board with photographs of the tangible material and used a marker to complete the task.

For the tangible condition, we made 12 houses, with dimensions of $6.5 \mathrm{~cm}$ high $\times 7 \mathrm{~cm}$ long, constructed of cardboard, foam plastic, ceramic tile and straw (see Figure 4). Then, for the pictorial condition, we took photographs of the 12 houses and printed them on a $70 \mathrm{~cm} \mathrm{x} 48 \mathrm{~cm}$ acrylic board. It is important to note that the photographs of the houses had the same measurements as the tangible houses.

We designed a post-test measuring the participants' level of retention in both groups. The test consists of 6 questions, each of which shows three photographs of houses, one correct and two incorrect. The test was administered to subjects in both groups, two months after the intervention.

The experiment compares the effectiveness of the use of tangible manipulatives, contrasted with photographs as pictorial representations of the same manipulative; the two learning conditions differ only in the medium of presentation. The experiment was to find several "hidden words" through a game. In total, there were three words that every subject had to discover. Three intervention protocols were developed, one for each hidden word. Each protocol consisted of a series of guidelines for interacting with the subject as well as a series of clues to help find the words.

Below are the three characteristics measured for each subject:

a. Time on task

b. Number of hints

c. Retention and consolidation of knowledge

\section{Results}

A Mann-Whitney U test was used to analyze the results. The group using tangible manipulatives reported an average time on task of 3:54 $\min (S D=72.4 \mathrm{sec})$. The group that worked with pictorial representations averaged 2:36 $\min (S D=48 \mathrm{seg})$. We conclude that sufficient evidence exists, requiring a confidence level of $=0.05$, that there is a significant difference between the two samples $(p=0.002)$. That is, subjects using pictorial representations spent less time on the task than those who worked with the tangible material.

The group using pictorial representations had an average of 2.0 hints $(S D=0.42)$. The group with tangible representations reported an average of 2.4 hints $(S D=0.53)$. We conclude that there is sufficient evidence, with a confidence of $=0.05$, that there is a significant difference between the two samples ( $p=$ $0.039)$. That is, subjects who worked with pictorial 
representations used a smaller number of hints for the task than those who worked with tangible representations.

According to the retention test, students who worked with pictorial representations had a score of $0.67(S D=0.20)$. As for tangible manipulation group, their average is $0.61(S D=0.19)$. We conclude that, taking the confidence of $=0.05$, there is insufficient evidence to conclude that there is a difference between the two samples $(p=0.337)$. That is, children who worked with pictorial representations received the same level of retention than those who worked with tangible representations. No significant difference was found in the retention of concepts.

\section{Conclusion and Discussion}

The goal of this paper was to compare the effects of physical manipulation on learning. The experimental conditions favored physical manipulation through the use of tangible material that children could play with, lifting, rotating, and, essentially, moving them to group them into clusters. The experimental condition B inhibited physical manipulation due to the use of photographic images rather than tangible material, and prevented spatial clustering of materials by replacing this with marks made with a marker.

The empirical evidence suggests that participants in the experimental condition A required more time and more help to complete the test. In addition, we found that both materials were equally effective for the consolidation of long-term information. By comparing the cognitive processes we can conclude that condition $\mathrm{B}$ allowed the participant to more effectively use their visual-spatial memory. In turn, the tangible material generated distraction in some way, and this caused a loss of attention and lack of memory when developing the test.

The result obtained for experimental condition B shows that, although involving less concrete representations, it allowed a better performance in this type of test. The photographs on the acrylic board were adequate symbolic objects and representations. Also, there is evidence that manipulatives are more attractive to children, and this causes some distraction. However, as they passed from one test to another, the subjects seemed to perform more evenly in time and number of hints used. In particular, the last attempt (test 3) may be highlighted here, where these two variables are very similar between the two experimental conditions. Indeed, it is possible that as the experience of the subjects of this test increases, the greater the likelihood that their performance would be similar, no matter what material they had used. However, these data need further investigation, since these are only trends that seem to arise.

In conclusion, it may be noted that the constructs from cognitive science theory are useful to explain learning through concrete and manipulative materials. In general, in the design of instructional materials, one must consider the cognitive processes described here: cognitive load, cognitive mapping, dual representation, and expertise. When explaining the empirical evidence, this framework can predict the performance of children, unlike the classical theories of cognitive development. According to the latter, the use of "concrete" is beneficial for young children who are at a particular level of thinking.

It has not been possible to find any effect of physical manipulation on children's learning. It is true that allowing physical activity with tangible objects increased the time taken on the task, which can be beneficial for learning. However, we also found the need for greater support for children to construct meaningful representations. Finally, if the consolidation of information in the memory is given to the same extent in both experimental conditions, one might assume that physical manipulation is not a variable that will improve learning in this population of children and for this type of activity.

\section{Suggestions and recommendations for further research}

It is very important to consider the characteristics of the population, such as socioeconomic level or cognitive development, which are key factors for analyzing the cognitive processes of children. Change in any of these variables may impact performance in such tests. Furthermore, when comparisons are made with different materials, it is important to pay attention to the way these are made and the instructions given for their use. In this case, the angle at which pictures of objects were taken should be taken into account, as they can alter the development of the test.

Finally, further research is necessary to expand the type of activities to study how physical manipulation can affect learning. It is also possible that if we decrease the age range of the subjects, the physical handling variable may be more relevant. So far, the presentation of the material seems to be of more importance than the type of interaction with it.

\section{Referencias}

Ainsworth, S. (2006). DeFT: A conceptual framework for considering learning with multiple representations. Learning and Instruction, 16, 183-198.

Ainsworth, S. (2008). The Educational Value of MultipleRepresentations when Learning Complex Scientific Concepts. En J. Gilbert, M. Reiner y M. Nakhleh, Visualization: Theory and Practice in Science Education (pp. 191-208). New York: Springer. 
Andrade Lotero, L. A., Cobo Charry, M. F., Díaz Díaz, L. N., Flórez Pineda, A. J., Garavito Muñoz, C., González Doblado, D. P., ... Villarraga-Acero, G. A. (2011). Manipulables Físicos para la Formación de Conceptos Artificiales en Niños de 6 a 8 años de Edad. Itinerario Educativo, 57, 157-183.

Artino, A. (2008). Cognitive load theory and the role of learner experience: An abbreviated review for educational practitioners. AACE Journal, 16, 425-439.

Bruner, J. (1965). El proceso mental en el aprendizaje. Madrid: Ediciones Narcea, S.A.

Clements, D. H. (1999). 'Concrete' manipulatives, concrete ideas. Contemporary Issues in Early Childhood, 1, 45-60.

Gauvain, M. (2001). Cultural Tools, Social Interaction and the Development of Thinking. Human Development, 44, 126-143.

Kalyuga, S., Ayres, P., Chandler, P. y Sweller, J. (2003). The Expertise Reversal Effect. Educational Psychologist, 38, 23-31.

Manches, A. y O'Malley, C. (in press). Tangibles for learning: a representational analysis of physical manipulation. $1-24$.

Martin, T. y Schwartz, D. L. (2005). Physically Distributed Learning: Adapting and Reinterpreting Physical Environments in the Development of Fraction Concepts. Cognitive Science, 29, 587-625.

Núñez, R. E., Edwards, L. D. y Matos, J. F. (1999). Embodied Cognition as Grounding for Situatedness and Context in Mathematics Education. Educational Studies in Mathematics, 39, 45-65.

Piaget, J. (1994). Seis estudios de psicología. Colombia: Editorial Labor.

Resnick, M., Martin, F., Berg, R., Borovoy, R., Colella, V., Kramer, K., y Silverman, B. (1998). Digital Manipulatives: New Toys to Think With. Paper presented at the Proceedings of the SIGCHI conference on Human Factors in computing systems, New York, NY, USA.

Robert, J. (1996). Elementary Statistics. Belmont, California: Wadsworth Publishing Company.

Scaife, M. y Rogers, Y. (1996). External cognition: How do graphical representations work? International Journal of Human-Computer Studies , 45, 185-213.

Sweller, J. (2002). Visualisation and Instructional Design. Retrieved 2010, from Knowledge Media Research
Center: http://www.iwm-kmrc.de/workshops/visualization/sweller.pdf

Tabbers, H. K., Martens, R. L. y van Merriënboer, J. J. (2004). Multimedia Instructions and Cognitive Load Theory: Effects of modality and cueing. British Journal of Educational Psychology, 74, 71-81.

Thornton, S. (1998). La Resolución Infantil de Problemas. Madrid: Ediciones Morata.

Uttal, D. H. (2003). On the Relation Between Play and Symbolic Thought, The Case of Mathematics Manipulatives. In O. N. Saracho y B. Spodek, Contemporary Perspectives on Play in Early Childhood Education (pp. 97-114). USA: Information Age Publishing.

Uttal, D. H., Liu, L. L. y DeLoache, J. S. (2003). Concreteness and Symbolic Development. In L. Balter, \& C. S. Tamis-LeMonda, Child Psychology: a Handbook of Contemporary Issues (pp. 197-184). New York: Psychology Press.

Uttal, D. H., Liu, L. L. y DeLoache, J. S. (1999). Taking a hard look at concreteness: Do concrete objects help young children to learn symbolic relations? In L. Balter, \& C. Tamis-LeMonda, Handbook of early child development (pp. 177-192). UK: Taylor \& Francis.

Uttal, D. H., Scudder, K. V. y DeLoache, J. S. (1997). Manipulatives as Symbols: A New Perspective on the Use of Concrete Objects to Teach Mathematics. Journal of Applied Developmental Psychology, 18, 37-54.

van Nimwegen, C., Burgos, D., van Oostendorp, H. y Schijf, H. (2006). The Paradox of the Assisted User: Guidance can be Counterproductive. Paper presented at the Proceedings of the SIGCHI conference on Human Factors in computing systems, New York, NY, USA.

Vygotsky, L. (1995). Pensamiento y Lenguaje. Buenos Aires: Ediciones Fausto.

Vygotsky, L. (1979). Desarrollo de los Procesos Psicológicos Superiores. Barcelona: Editorial Crítica.

Wood, D. (2000). Cómo piensan y aprenden los niños. México: Siglo Veintiuno Editores.

Zuckerman, O., Arida, S. y Resnick, M. (2005). Extending Tangible Interfaces for Education: Digital Montessoriinspired Manipulatives. Paper presented at the Proceedings of the SIGCHI conference on Human Factors in computing systems, New York, NY, USA.

Manuscrito recibido: 18/06/2011

Revisión recibida: 23/11/2011

Manuscrito aceptado: 21/12/2011 